\title{
Análisis de estrategias de autorregulación en estudiantes de pedagogía de una universidad chilena
}

\author{
Carolina Zambrano-Matamala ${ }^{1 *}$, Alejandro Díaz-Mujica ${ }^{2}$, María V. Perez-Villalobos² y Darío Rojas-Díaz ${ }^{1}$ \\ (1) Departamento de Metodología de Investigación e Informática Educacional, Facultad de Educación \\ Universidad de Concepción, Concepción, Chile. (correo-e: carolinazambrano@gmail.com; dfrojas@gmail.com) \\ (2) Departamento de Psicología, Facultad de Ciencias Sociales, Universidad de Concepción, Concepción, Chile. \\ (correo-e: adiazm@udec.cl; marperez@udec.cl)
}

* Autor a quien debe ser enviada la correspondencia.

Recibido Feb. 17, 2020; Aceptado Abr. 18, 2020; Versión final Jun. 29, 2020; Publicado Oct. 2020

\begin{abstract}
Resumen
El objetivo principal de este estudio fue analizar las estrategias de autorregulación medidas desde la perspectiva del modelo de Zimmerman y desde cuatro dimensiones: i) hábitos inadecuados de regulación; ii) organización del entorno; iii) búsqueda de información; y iv) organización de la tarea. La población consistió de 322 estudiantes de primer año de carreras de pedagogía en Chile. Los datos se analizaron mediante estadísticos descriptivos de confiablidad y diferencia de medias. Los resultados evidencian bajas puntuaciones para todas las dimensiones de las estrategias de autorregulación. Los hallazgos indican diferencias estadísticamente significativas entre estudiantes provenientes de establecimientos de administración municipal y particular subvencionado para la dimensión hábitos inadecuados de regulación. Para esta misma dimensión, también se encontraron diferencias estadísticamente significativas entre establecimientos municipales y establecimientos particulares. En conclusión, los resultados aportan evidencia empírica respecto a la diferenciación de los estudiantes que provienen de establecimientos municipales y muestra la importancia del fomento de las estrategias de autorregulación.
\end{abstract}

Palabras clave: estrategias de autorregulación; aprendizaje autorregulado; enseñanza superior; segmentación social

\section{Analysis of self-regulation strategies in pedagogy students from a Chilean university}

\begin{abstract}
The main objective of the present study was to analyze self-regulation strategies from the perspective of the Zimmerman model and from four dimensions: 1) inadequate regulatory habits, 2) environment organization, 3 ) information search, and 4) task organization. The population consisted of 322 first-year pedagogy undergraduate students from Chile. Data analyzes were performed using descriptive statistics, reliability, and difference of means. The results showed low scores for all dimensions of self-regulation strategies. There were statistically significant differences between students coming from municipal public high schools and students coming from subsidized private high schools for the inadequate regulation habit. This dimension was also found to be statistically different between municipal high schools and private high schools. In conclusion, the results provide empirical evidence about the social segmentation of undergraduate students coming from public high schools and show the importance of promoting self-regulation strategies.
\end{abstract}

Keywords: self-regulation strategies; self-regulated learning; higher education; social segmentation 


\section{INTRODUCCIÓN}

El concepto autorregulación del aprendizaje (ARA) ha sido definido por diferentes autores, entre los que destacan Boekaerts (1995), Pintrich y De Groot (1990), Winne (1996), Zimmerman (1989), entre otros. Al respecto, el consenso entre las distintas aproximaciones sugiere que ARA es el proceso por el cual un estudiante puede gestionar sus cogniciones, comportamientos y emociones para mejorar su capacidad y eficiencia asociada al rendimiento académico esperado. Por ello, en los últimos años diferentes estudios coinciden en que ARA es un factor relevante en el nivel de rendimiento académico de los estudiantes (Díaz, Pérez, Valenzuela, Muñoz, Rivas y Salas, 2010; Hernández y Camargo, 2017b; Mega, Ronconi y de Beni, 2014; entre otros). También es importante mencionar que ARA es un proceso complejo, tanto estructural como procesual (Zimmerman, 1989). Por ello, debido a la complejidad de este constructo se hace necesario especificar qué variable y/o proceso es el que se desea analizar con tal de utilizar instrumentos que permitan medir de forma válida y fiable la variable en estudio (Streiner, Norman y Cairney, 2015). Además, es necesario comprender conceptualmente la variable que se desea medir respecto de ARA (Bransen y Govaerts, 2020).

En este contexto, el presente estudio mide estrategias de autorregulación debido a que esta variable de ARA representa un componente clave en los procesos de aprendizaje (Elvira-Valdés y Pujol, 2012). Asimismo, se ha señalado que "el uso de estrategias de autorregulación provee a los estudiantes un mayor control sobre su propio proceso de aprendizaje en la adquisición de conocimientos y destrezas" (Elvira-Valdés y Pujol, 2012, p.368). También, diversos estudios señalan que las estrategias de autorregulación se relacionan favorablemente con el rendimiento académico desde la perspectiva del modelo de Zimmerman (Cleary y Platten, 2013).

En suma, las estrategias de autorregulación son necesarias para el estudiante universitario porque quienes las aplican pueden llegar a ser capaces de planificar, monitorear y evaluar el proceso de aprendizaje en la consecución de objetivos de aprendizaje (Pool-Cibrián y Martínez-Guerrero, 2013). En efecto, la importancia de estudiar las estrategias de autorregulación en los estudiantes de primer año de universidad, en carreras del área de educación, se basa en la necesidad de poder contar con un diagnóstico sistémico que pueda ayudar a crear planes y/o programas de acción adecuados a las instituciones (Díaz-Mujica, Pérez-Villalobos, González-Pienda y Núñez-Pérez, 2017), porque ARA es una capacidad que se puede entrenar (Boekaerts y Cascallar, 2006).

Otro punto relevante ha destacar es que existe una baja cantidad de investigaciones que miden estrategias de autorregulación en estudiantes de pedagogía en Latinoamérica (Hernández y Camargo, 2017b) y especialmente en Chile. Además, en el contexto de las habilidades del siglo XXI que son promovidas por Framework for 21st Century Learning (2019) el uso de estrategias de autorregulación es una variable clave para ARA sobre todo en estudiantes de pedagogía que tienen el doble rol de por un lado ser un estudiante que se autorregula y por otro lado un futuro profesor que debe aplicar las estrategias de autorregulación en su propia labor docente (Zambrano-Matamala, Rojas-Diaz, Salcedo-Lagos, Albarrán-Torres y Díaz-Mujica, 2019).

Sobre la base de los antecedentes anteriores, el objetivo principal del estudio es analizar las estrategias de autorregulación medidas a través de cuatro dimensiones: 1) Organización del entorno, 2) Organización de la tarea, 3) Búsqueda de información y 4) Hábitos inadecuados de regulación, estas dimensiones fueron definidas por (Hernández y Camargo, 2017a) en el instrumento SRSI-SR adaptado al español y a estudiantes universitarios colombianos. La validación de este instrumento para población de estudiantes universitarios chilenos de carreras del área de la educación se realizó en Chile (Zambrano, Rojas, Díaz y Salcedo, 2018) corroborando la estructura de cuatro dimensiones propuesta por Hernández y Camargo (2017a). Además, como objetivo secundario se compara las estrategias de autorregulación medidas a través de las cuatro dimensiones con dos variables sociodemográficas que son: tipo de establecimiento educacional y género.

La variable tipo de establecimiento educacional se refiere al establecimiento de proveniencia de la enseñanza secundaria que es relevante en el sistema educativo chileno debido a la alta segmentación social en: alto (Bellei, Orellana y Canales, 2020), medio (Canales, Bellei y Orellana, 2016) y bajo (Bellei, Canales, Orellana y Contreras, 2017). Es decir, los estudiantes cursan su educación primaria y secundaria en establecimientos clasificados como: Municipal, Particular Subvencionado o Particular dependiendo de la capacidad de pago de sus padres. En este sentido, investigadores han reportado evidencia que indica que el grupo social de los estudiantes (alto, medio y bajo) es equiparable al tipo de dependencia administrativa de los establecimientos educacionales de enseñanza primaria y secundaria (Municipal, Particular Subvencionado, Particular) producto de las características de segmentación del propio sistema educativo chileno (Bellei, Orellana y Canales, 2020; Valenzuela, Bellei y De los Ríos, 2014) y la reproducción social (Bourdieu y Passeron, 2009) que se genera desde la estructura del sistema de educación chileno. 


\section{MÉTODO}

La investigación tiene un enfoque metodológico cuantitativo de diseño no experimental, transversal, descriptivo y comparativo (Ato, López y Benavente, 2013).

\section{Participantes}

Se encuestó a 322 estudiantes pertenecientes a seis especialidades: Educación General Básica, Educación Diferencial, Educación Parvularia, Pedagogía en Historia y Geografía, Pedagogía en Educación Física y Pedagogía en Matemática. El 39,09 \% de los participantes son hombres y el $60.91 \%$ son mujeres. La Tabla 1 presenta la muestra agrupada por Carrera y Tipo de establecimiento educacional secundario de procedencia (Municipal, Particular Subvencionado, Particular), edad media y desviación típica.

Es importante mencionar que los 21 estudiantes provenientes de establecimientos particulares que se observan en la Tabla 1 son reflejo de la característica de segregación del sistema educativo chileno (Bellei, Orellana y Canales, 2020; Canales, Bellei y Orellana, 2016; Valenzuela, Bellei y de los Ríos, 2014). Esta característica hace referencia a la alta segregación del sistema educativo chileno, en el cual sólo entre el $6 \%$ y $7 \%$ de los estudiantes asisten a un establecimiento particular pagado (Bellei, Orellana y Canales, 2020). Para la selección de los participantes se utilizó la técnica de muestreo no probabilístico por conveniencia, debido a que se escogió de forma intencionada estudiantes que cursaran primer año de carreras de pedagogía de una facultad de educación en una universidad de Chile.

Tabla 1: Estadísticos descriptivos de la muestra.

\begin{tabular}{|l|l|l|l|l|l|}
\hline Carrera & \multicolumn{4}{l|}{ Edad } & \multicolumn{4}{l|}{ Establecimiento } \\
\hline & $M$ (Media) & $\begin{array}{l}\text { SD (Desviación } \\
\text { Estándar) }\end{array}$ & Municipal & $\begin{array}{l}\text { Particular } \\
\text { Subvencionado }\end{array}$ & Particular \\
\hline Educación General Básica & 2.2165 & 0.480 & 42 & 37 & 8 \\
\hline Educación Diferencial & 2.1277 & 0.745 & 14 & 23 & 0 \\
\hline Educación Parvularia & 2.0945 & 0.223 & 16 & 23 & 4 \\
\hline Pedagogía en Historia y Geografía & 2.2183 & 0.451 & 23 & 22 & 2 \\
\hline Pedagogía en Educación Física & 2.1100 & 0.679 & 15 & 40 & 7 \\
\hline Pedagogía en Matemática & 2.1720 & 0.436 & 16 & 30 & 0 \\
\hline Totales: & 2.1565 & & 126 & 175 & 21 \\
\hline
\end{tabular}

\section{Procedimiento}

Los estudiantes responden el instrumento SRSI-SR adaptado (Hernández y Camargo, 2017a) en grupos de no más de 25 personas en sesiones de 20 minutos. La aplicación se llevó a cabo durante el primer y segundo semestre en laboratorios de computación de una facultad de educación de una universidad chilena. La respuesta al instrumento se realiza de forma voluntaria y con consentimiento informado.

\section{Instrumento y variables}

Se utilizó la escala SRSI-SR adaptada (Hernández y Camargo, 2017a) para estudiantes universitarios que está compuesta por 4 dimensiones. Las dimensiones que da cuenta son: 1) Organización del Entorno (OE): se refiere a las acciones anticipadas o realizadas por el individuo encaminadas a disminuir las variables distractoras, que comprometen la definición y ocupación de un sitio de estudio, y la priorización de las actividades académicas respecto a actividades alternativas no académicas (sociales o no sociales). 2) Búsqueda de Información (BI): se define como el conjunto de acciones deliberadas orientadas a la identificación y uso de fuentes de información necesarias para el desarrollo de una actividad académica. 3) Organización de la tarea (OT): es la planificación de la secuencia de acciones requeridas para la realización de una actividad académica a partir de la precisión de un estado meta y la identificación de los recursos disponibles. 4) Hábitos Inadecuados de Regulación (HIR): es definido como el conjunto de conductas evitativas del individuo respecto al cuidado e involucramiento activo en actividades de aprendizaje académico (Hernández y Camargo, 2017a).

Para las respuestas a cada ítem del instrumento se utilizó una puntuación de 1 a 5 en escala de Likert con las siguientes valoraciones (nunca, casi nunca, regularmente, casi siempre, siempre). Los ítems y alpha de Cronbach a calculado para cada dimensión se presentan en la Tabla 2. En ésta las dimensiones e ítems fueron adaptados de Hernández y Camargo (2017a) y los cálculos de alpha de Cronbach fueron elaborados por los autores. 
Tabla 2: Dimensiones, ítems y alpha de Cronbach asociados a cada dimensión del instrumento (adaptado de Hernández y Camargo 2017a)

\begin{tabular}{|l|c|}
\hline Ítems y Dimensiones & $\alpha$ \\
\hline Dimensión Hábitos Inadecuados de Regulación (HIR) & .88 \\
\hline 1.Evito preguntar en clase cuando no entiendo el tema & \\
\hline 2.Me rindo fácilmente cuando no entiendo algo & \\
\hline 3.Cuando estoy estudiando ignoro los temas que son difíciles de entender & \\
\hline 4.Permito que las personas me interrumpan cuando estoy estudiando & .86 \\
\hline Dimensión Organización del Entorno (OE) & \\
\hline 5.Intento estudiar en un sitio tranquilo & \\
\hline 6.Intento estudiar en un lugar sin distracciones (ruido, gente hablando) & \\
\hline 7.Me aseguro de que nadie me distraiga cuando estoy estudiando & \\
\hline 8.Termino todas mis actividades académicas antes de iniciar otro tipo de actividades & .89 \\
\hline Dimensión Búsqueda de Información (BI) & \\
\hline 9.Cuando no comprendo algún tema le pregunto al profesor & \\
\hline 10.Realizo búsquedas bibliográficas adicionales que me ayuden a comprender los temas de clase & \\
\hline 11.Busco material complementario de los temas vistos en clase & .87 \\
\hline 12. Investigo cuando no entiendo algo sobre las tareas que me asignan & \\
\hline Dimensión Organización de la Tarea (OT) & \\
\hline 13.Planifico en qué orden realizaré mis actividades académicas & \\
\hline 14.Coordino mi tiempo de acuerdo con las actividades académicas asignadas & \\
\hline 15.Hago un horario para organizar mi tiempo de estudio & \\
\hline 16.Uso algún método para mantener en orden el material de mis clases & \\
\hline
\end{tabular}

Para la validación interna de cada dimensión se calculó el alpha de Cronbach, considerando valores $\alpha>.80$ como fiables (Hu y Bentler, 1999). En este sentido, en la Tabla 2 se observa que todas las dimensiones presentan valores $\alpha>.80$. Además, la consistencia interna del instrumento global es alta $(\alpha=.88)$. Por otro lado, la literatura recomienda emplear múltiples indicadores para evaluar el ajuste del modelo (Hu y Bentler, 1999). Se destacan el índice de ajuste comparativo (CFI), el índice de bondad de ajuste (GFI) y el error cuadrático medio de aproximación (RMSEA). Por ello, las propiedades psicométricas del instrumento para población de estudiantes chilenos de carreras del área educación se revisaron a través de un análisis factorial confirmatorio (AFC) que se reporta en Zambrano et al. (2018) donde se corrobora la estructura de cuatro dimensiones (factores) propuesta en (Hernández y Camargo, 2017a).

Finalmente, la aplicación de AFC obtuvo un modelo con buen ajuste para los índices RMSEA, SRMR, GFI, AGFI y para los índices comparativos NFI, IFI, CFI y AIC se obtuvo el mejor ajuste cuyos valores se observan en la Tabla 3. Los resultados de buen y mejor ajuste para los índices significan un ajuste satisfactorio entre las estructuras teóricas y los datos empíricos recogidos por la aplicación del instrumento SRSI-SR adaptado. Por ello, el instrumento es recomendable para intervenciones en aula.

Tabla 3: Índices de ajuste

\begin{tabular}{|l|l|l|l|l|l|l|l|}
\hline RMSEA & SRMR & GFI & AGFI & NFI & IFI & CFI & AIC \\
\hline .071 & .070 & .930 & .891 & .910 & .939 & .938 & 236.631 \\
\hline
\end{tabular}

Cabe señalar que el procedimiento de AFC aplicado en (Zambrano et al., 2018) permitió evaluar la validez y fiabilidad de cada ítem (Streiner, Norman y Cairney, 2015). Por otro lado, las variables sociodemográficas que se analizan son: género y tipo de establecimiento (Municipal, Particular Subvencionado y Particular) al que puede asociarse el grupo social del estudiante (bajo, medio y alto) (Bellei, Canales, Orellana y Contreras, 2017; Bellei, Orellana y Canales, 2020; Valenzuela, Bellei y de los Ríos, 2014).

\section{Análisis de Datos}

En primera instancia se realizó un análisis estadístico descriptivo para cada dimensión y carrera usando media (M) y desviación estándar (SD). Después, se procedió a realizar comparaciones entre las carreras aplicando la prueba no paramétrica de Kruskal-Wallis con pruebas post-hoc usando Dunn-Bonferroni. También, se buscó diferencias significativas entre las dimensiones de estrategias de autorregulación por género y diferencias significativas entre tipos de establecimiento de procedencia (Municipal, Particular Subvencionado y Particular). Para la validación interna del instrumento se calculó el alpha de Cronbach $\alpha$. 


\section{RESULTADOS}

Se presenta el análisis estadístico descriptivo e inferencial de los datos recopilados en base a las 4 dimensiones para medir estrategias de autorregulación. La Tabla 4 muestra los estadísticos descriptivos de cada dimensión por carrera. Se observa, que la carrera de Pedagogía en Educación Física presenta la valoración más baja para la dimensión Hábitos Inadecuados de Regulación (HIR) con $M=1.961$. Al contrario, Educación General Básica M=3.076 tiene la valoración más alta para la dimensión HIR junto a Pedagogía en Historia y Geografía M=3.074.

El mejor puntaje para la dimensión Organización del Entorno $(\mathrm{OE})$ lo obtiene Educación Parvularia M=2.586. Por otro lado, las menores valoraciones para esta dimensión las obtuvieron Educación General Básica M= 2.010 y Pedagogía en Historia y Geografía M=2.089. En el caso de la dimensión Búsqueda de Información (BI), en todas las carreras la puntuación es baja debido a que la puntuación máxima podría llegar a 5. Para la dimensión Organización de la Tarea (OT) la mejor puntuación es para Educación Diferencial junto a pedagogía en Matemática. No obstante, en general las puntuaciones de OT son similares en todas las carreras. Los resultados anteriores sugieren que se debe impulsar el desarrollo de la capacidad de uso de estrategias de autorregulación en estudiantes de pedagogía de forma sistemática y desde el aula, creando procesos consientes de reflexión e importancia de las acciones de autorregulación.

Tabla 4: Estadísticos descriptivos para cada dimensión y carrera. Los valores corresponden a (M) y (SD) de la suma para cada dimensión

\begin{tabular}{|c|c|c|c|c|c|c|c|c|}
\hline \multirow[t]{2}{*}{ Carreras } & \multicolumn{2}{|l|}{$H I R$} & \multicolumn{2}{|l|}{$O E$} & \multicolumn{2}{|l|}{$B I$} & \multicolumn{2}{|l|}{ OT } \\
\hline & $M$ & $S D$ & $M$ & $S D$ & $M$ & $S D$ & $M$ & $S D$ \\
\hline Educación General Básica & 3.076 & 0.694 & 2.010 & 0.902 & 1.825 & 0.336 & 1.955 & 0.227 \\
\hline Educación Diferencial & 2.118 & 0.844 & 2.340 & 0.596 & 1.873 & 0.809 & 2.180 & 0.770 \\
\hline Educación Parvularia & 2.110 & 0.764 & 2.586 & 0.282 & 1.627 & 0.735 & 2.055 & 0.712 \\
\hline Pedagogía en Historia y Geografía & 3.074 & 0.878 & 2.089 & 0.586 & 1.719 & 0.965 & 1.991 & 0.511 \\
\hline Pedagogía en Educación Física & 1.961 & 0.485 & 2.480 & 0.912 & 1.945 & 0.912 & 2.058 & 0.343 \\
\hline Pedagogía en Matemática & 2.306 & 0.884 & 2.229 & 0.134 & 1.985 & 0.328 & 2.163 & 0.415 \\
\hline
\end{tabular}

Después del análisis estadístico descriptivo por carrera se procedió a realizar una comparación entre las carreras cuyos resultados se observan en la Tabla 5. En la comparación de carreras se encontraron diferencias estadísticamente significativas entre las medianas de las dimensiones $\mathrm{HIR}(\mathrm{H}(5)=77.888, p<.001)$, $\mathrm{OE}(\mathrm{H}(5)=30.381, p<.001)$ y BI $(\mathrm{H}(5)=32.283, p<.001)$. Para el cálculo del estadístico H se utilizó la prueba no paramétrica de Kruskal-Wallis. Luego, las pruebas post-hoc usando Dunn-Bonferroni para las dimensiones que presentaron diferencias significativas (HIR, OE y BI) entre las carreras se muestran en la Tabla 5. En ella se observa, por cada pareja de carreras comparadas, las dimensiones que presentaron diferencia significativa con $p<.05$.

Tabla 5: Comparación de carreras y diferencias significativas entre pares de ellas por cada dimensión según las pruebas post-hoc utilizando la prueba de Dunn-Bonferroni

\begin{tabular}{|l|l|l|l|l|l|}
\hline Carreras & $\begin{array}{l}\text { Educación } \\
\text { General Básica }\end{array}$ & $\begin{array}{l}\text { Educación } \\
\text { Diferencial }\end{array}$ & $\begin{array}{l}\text { Educación } \\
\text { Parvularia }\end{array}$ & $\begin{array}{l}\text { Pedagogía en } \\
\text { Historia y } \\
\text { Geografía }\end{array}$ & $\begin{array}{l}\text { Pedagogía en } \\
\text { Educación } \\
\text { Física }\end{array}$ \\
\hline Educación General Básica & - & - & - & - & - \\
\hline Educación Diferencial & HIR, OE & - & - & - & - \\
\hline Educación Parvularia & HIR, BI & OE, BI & - & - & - \\
\hline $\begin{array}{l}\text { Pedagogía en Historia y } \\
\text { Geografía }\end{array}$ & & HIR, BI & HIR, OE & - & - \\
\hline $\begin{array}{l}\text { Pedagogía en Educación } \\
\text { Física }\end{array}$ & HIR & & & HIR & - \\
\hline Pedagogía Matemática & HIR, OE & & HIR, BI & HIR, OE & HIR, OE \\
\hline
\end{tabular}

En la comparación por género, la prueba realizada para comprobar la existencia de una diferencia significativa en estos grupos reveló que sólo hay diferencias significativas entre el género femenino y masculino para la dimensión OE $(Z=-2.081, p<.05)$, mientras que para HIR, BI y OT no existe diferencia significativa entre géneros con $(Z=.039, p=.969),(Z=-.749, p=.54)$ y $(Z=-.976, p<.329)$ respectivamente. Los estadísticos descriptivos para cada dimensión y género se presentan en la Tabla 6. 
Tabla 6: Estadísticos descriptivos para cada dimensión, género y tipo de establecimiento. Los valores corresponden a media $(\mathrm{M})$ y desviación estándar (SD) de la suma de ítems para cada dimensión.

\begin{tabular}{|l|l|l|l|l|l|l|l|l|l|l|}
\hline & \multicolumn{2}{|l|}{$\begin{array}{l}\text { Establecimiento } \\
\text { Municipal }\end{array}$} & \multicolumn{2}{l|}{$\begin{array}{l}\text { Establecimiento } \\
\text { Particular } \\
\text { Subvencionado }\end{array}$} & \multicolumn{2}{l|}{$\begin{array}{l}\text { Establecimiento } \\
\text { Particular } \\
\text { Pagado }\end{array}$} & \multicolumn{2}{l|}{ Femenino } & \multicolumn{2}{l|}{ Masculino } \\
\hline Dimensiones & $M$ & $S D$ & $M$ & $S D$ & $M$ & $S D$ & $M$ & $S D$ & $M$ & $S D$ \\
\hline HIR & 3.523 & 0.703 & 1.984 & 0.531 & 1.819 & 0.081 & 2.364 & 0.608 & 2.532 & 0.701 \\
\hline OE & 1.441 & 0.826 & 2.510 & 0.137 & 2.914 & 0.502 & 2.413 & 0.944 & 2.164 & 0.019 \\
\hline BI & 1.528 & 0.894 & 1.875 & 0.544 & 2.105 & 0.964 & 1.825 & 0.586 & 1.829 & 0.773 \\
\hline OT & 1.723 & 0.351 & 2.141 & 0.017 & 2.376 & 0.376 & 2.145 & 0.147 & 1.968 & 0.141 \\
\hline
\end{tabular}

Respecto a los estadísticos descriptivos relacionados a los tipos de establecimiento de procedencia estos se presentan en la Tabla 6 para cada dimensión calculada. En ésta se observa que los estudiantes que provienen de establecimientos municipales presentan valoraciones bajas para las dimensiones OE, BI y OT. Para la dimensión HIR los estudiantes de establecimiento municipal presentan una valoración alta $M=3.523$ lo que significa que evitan preguntar en clase cuando no entienden el tema, se rinden fácilmente cuando no entienden algo, cuando estudian ignoran los temas que son difíciles de entender y permiten que las personas interrumpan su estudio, mientras que los estudiantes de establecimientos particular pagado presentan mejores valoraciones con medias mayores a 3 para las dimensiones OE, BI y OT que significa autovaloración de la regularidad.

Al comparar entre los tipos de establecimiento, los hallazgos muestran diferencias estadísticamente significativas sólo para la dimensión $\mathrm{HIR}(\mathrm{H}(2)=12.240, p<.005)$, mientras que para $\mathrm{OE}(\mathrm{H}(2)=2.348, p=.309)$, $\mathrm{BI}(\mathrm{H}(2)=.927, p=.629)$ y OT $(\mathrm{H}(2)=4.857, p=.088)$ no se encontró diferencias estadísticamente significativas. Las pruebas post-hoc usando Dunn-Bonferroni para la dimensión HIR presentó diferencias significativas entre estudiantes de Establecimiento Municipal y Establecimiento Particular Subvencionado, y entre el Establecimiento Municipal y Establecimiento Particular Pagado. Sin embargo, entre los dos tipos de establecimientos particulares no se encontró diferencia significativa. Esto sugiere que, entre los tipos de establecimientos, la procedencia del estudiante desde establecimiento de administración municipal es diferente a los otros dos tipos de establecimiento respecto a presentar hábitos inadecuados de regulación.

En este sentido, en Chile entre el $7 \%$ al $8 \%$ de los estudiantes asiste a un establecimiento particular pagado. Al contrario, el $93 \%$ asiste a establecimiento municipal o a establecimiento particular subvencionado lo que genera diferencias en la estructura social chilena y como consecuencia segrega a la población desde la educación con diferencias que etiquetan a las personas y mantienen la vulnerabilidad de los estratos más bajos (Bellei, Orellana y Canales, 2020). Cabe señalar que en los últimos años los establecimientos de tipo particular subvencionado han aumentado su matrícula porque los apoderados de sector económico medio de Chile los prefieren ya que obtienen mejores resultados en las mediciones estandarizadas y para mantenerse en un grupo socioeconómico medio (Bellei, Orellana y Canales, 2020; Canales, Bellei y Orellana, 2016; Valenzuela, Bellei y de los Ríos, 2014).

\section{DISCUSION}

Uno de los principales hallazgos permitió evidenciar diferencias estadísticamente significativas entre estudiantes provenientes de establecimiento municipal y establecimiento particular subvencionado. Asimismo, se encontró diferencias estadísticamente significativas entre estudiantes provenientes de establecimiento municipal y de establecimiento particular. En ambos casos las diferencias se reportan sólo para la dimensión hábitos inadecuados de regulación.

Los resultados anteriores aportan datos a la situación de desigualdad educativa en Chile estudiada por Bellei, Orellana y Canales (2020), Canales, Bellei y Orellana (2016) y Valenzuela, Bellei y De los Ríos (2014) quienes señalan que la segregación del sistema educativo en Chile provoca efectos negativos en los resultados de aprendizaje de los estudiantes principalmente de establecimientos municipales. Por ello, los establecimientos de tipo particular subvencionado han aumentado su matrícula porque los apoderados de sector económico medio de Chile los prefieren ya que reportan mejores indicadores en las pruebas estandarizadas de chile (Canales, Bellei y Orellana, 2016). Esta situación no ha cambiado y continua la segregación estructural del sistema educativo chileno (Bellei, Orellana y Canales, 2020) ayudando a la reproducción social desde la escuela (Bourdieu y Passeron, 2009). Porque el papel de la educación en la reproducción social se refiere al grado en que el capital cultural familiar determina los desempeños académicos y la acumulación de capital cultural en general, usando la justificación del talento o mérito individual demostrado en el proceso educativo, legitimando así una determinada posición de clase (Bourdieu y Passeron, 2009). 
La situación de desigualdad descrita anteriormente se ha mantenido en los últimos años en chile, ya que continua un sistema educacional altamente segregado (Valenzuela, Bellei y de los Ríos, 2014; Bellei, Orellana y Canales, 2020) donde los establecimientos de administración municipal presentan resultados académicos insuficientes respecto de los aprendizajes necesarios para un estudiante. Por eso, se sugiere la conveniencia de enseñar y fomentar el uso de estrategias de autorregulación desde el currículum de los estudiantes universitarios como ha sido planteado en (Daura, 2017; Peeters, De Backer, Reina, Kindekens, Buffel y Lombaerts, 2014; Zambrano, 2016; entre otros). Especialmente como intervenciones en aula que permitan de forma sistemática medir las variables en estudio respecto del aprendizaje esperado y así eviten la deserción universitaria de los estudiantes menos favorecidos. No obstante, el desafío real es repensar la estructura del sistema educativo chileno.

En relación con los resultados de las puntuaciones obtenidas sin distinguir por carreras, la dimensión HIR presenta una alta valoración. Esto indica que los estudiantes participes del estudio no regulan "el conjunto de conductas evitativas respecto al cuidado e involucramiento activo en actividades de aprendizaje académico" (Hernández y Camargo, 2017a, p.15). En el caso de las dimensiones BI, OE y OT respecto del total de la muestra, sin distinguir ni por carrera ni por género, las medias son bajas. Esto indicaría que los estudiantes no son constantes en el uso de estas estrategias. En el caso de la dimensión OE, se sugiere que los estudiantes no siempre realizan acciones anticipadas o en el momento mismo de la ejecución del aprendizaje para disminuir la influencia de variables distractoras. Es decir, no es constante "la priorización de las actividades académicas respecto a actividades alternativas no académicas" (Hernández y Camargo, 2017a, p.15).

En el caso de la dimensión BI que representa "el conjunto de acciones deliberadas orientadas a la identificación y uso de fuentes de información necesarias para el desarrollo de una actividad académica" (Hernández y Camargo, 2017a, p.15), se sugiere que el comportamiento declarado tampoco es constante, sino que son acciones realizadas ocasionalmente. Lo anterior también coincide con el resultado de la dimensión OE. La dimensión OT que se conceptualiza como "la planificación de la secuencia de acciones requeridas para la realización de una actividad académica a partir de la precisión de un estado meta y la identificación de los recursos disponibles" (Hernández y Camargo, 2017a, p.15), obtiene puntuaciones medias que sugieren que las acciones de los estudiantes en este sentido no son constantes, lo que puede influir de forma negativa sobre su proceso de aprendizaje.

En relación con otros estudios que miden estrategias de autorregulación los resultados coinciden con los hallazgos de bajas puntuaciones en estrategias de autorregulación (Fernández, Bernardo, Suárez, Cerezo, Núñez y Rosario, 2013). Fernández et al. (2013) muestran que los resultados de un análisis de regresión jerárquica evidencian que el uso de estrategias de autorregulación del aprendizaje depende mayoritariamente de la capacidad percibida para ello y de otras variables personales como el interés por aprender y la utilidad percibida del uso de dichas estrategias, situación que refuerza la importancia del entrenamiento de esta variable de ARA para el éxito académico de los estudiantes más desfavorecidos.

En suma, los hallazgos reportados en esta investigación aportan a la escasa evidencia empírica que se ha reportado en Latinoamérica (Hernández y Camargo, 2017b) y especialmente en Chile respecto de esta variable de ARA. Además, como proyección de los resultados es necesario implementar acciones pedagógicas y didácticas para el fomento de las estrategias de autorregulación medidas a través de las dimensiones HIR, BI, OE y OT u otras (Peeters et al., 2014; Zambrano, 2016; Daura, 2017, entre otros). En este sentido, los hábitos inadecuados de regulación HIR se pueden mejorar desde el aula facilitando experiencias que permitan a los estudiantes participar en el desarrollo de actividades complejas usando por ejemplo estrategia ABP. Asimismo, incorporar la búsqueda de información en el desarrollo del ABP. De este modo, el profesor puede planificar el desarrollo del proyecto ABP incorporando las cuatro dimensiones y al mismo tiempo requerir que el estudiante y su grupo construyan una planificación estratégica del proyecto ABP que incorpore las cuatro dimensiones para que tome conciencia de su proceso de aprendizaje y lo aplique.

Las limitaciones del estudio están asociadas al tipo de diseño que se utilizó, que no permite establecer conclusiones de causalidad entre las variables estudiadas. No obstante, como proyección se considera realizar estudios longitudinales prospectivos usando modelos de ecuaciones estructurales para analizar causalidad e incorporar variables como resiliencia y autoconcepto. También, sería interesante evaluar las distintas escalas de medición de la variable estrategias de autorregulación como las usadas en (Boruchovitch y Santos, 2015; Fernández et al., 2013). 


\section{CONCLUSIONES}

El objetivo principal del estudio fue analizar las estrategias de autorregulación medidas a través de cuatro dimensiones: OE, OT, BI y HIR. En este sentido, se concluye que:

1.- Las puntuaciones para las dimensiones HIR y OE son cercanas a la media y para las dimensiones $\mathrm{BI}$ y OT se observa una baja puntuación en todas las carreras lo que advierte un comportamiento no regular en la aplicación de estrategias de autorregulación.

El objetivo secundario fue comparar las estrategias de autorregulación medidas a través de las cuatro dimensiones con dos variables sociodemográficas: tipo de establecimiento educacional y género. En este contexto, se concluye que:

2.- Existen diferencias estadísticamente significativas entre estudiantes provenientes de establecimiento municipal con estudiantes provenientes de establecimiento particular subvencionado y particular pagado respecto de la dimensión HIR.

3.- Se sugiere el desarrollo de procesos de enseñanza aprendizaje que incluyan actividades complejas. Por ejemplo, incluir la estrategia ABP desde el aula para fomentar la aplicación de estrategias de autorregulación del aprendizaje.

En suma, los resultados aportan evidencia empírica respecto a la diferenciación de los estudiantes que provienen de establecimientos municipales y muestra la importancia del fomento de las estrategias de autorregulación desde la perspectiva del modelo de Zimmerman en el contexto actual del sistema educativo. No obstante, el desafío es repensar la estructura del sistema educativo chileno a la luz de las demandas sociales y del contexto de incertidumbre que ha provocado la pandemia por covid-19.

\section{AGRADECIMIENTOS}

Esta investigación es parte de la tesis de Doctor en Educación de Carolina Zambrano. Se realizó como parte del Proyecto de investigación más amplio, CONICYT/FONDECYT 1161502, Modelo explicativo de la permanencia y el abandono de los estudios universitarios, basado en procesos cognitivo motivacionales.

\section{REFERENCIAS}

Anthonysamy, L.N., Koo, A.C., y Hew, S.H., Self-regulated learning strategies in higher education: Fostering digital literacy for sustainable lifelong learning. doi:10.1007/s10639-020-10201-8, Educ Inf Technol, 2020, 1-22 (2020).

Ato, M.A, López-García, J.J., y Benavente, A.A., A classification system for research designs in psychology. doi: 10.6018/analesps.29.3.178511, Anales de Psicología, 29(3), 1038-1059 (2013)

Bellei, C., Orellana, V., y Canales, M., Elección de escuela en la clase alta chilena. Comunidad, identidad y cierre social. doi:10.14507/epaa.28.3884, Archivos Analíticos de Políticas Educativas, 28(5), 1-22 (2020)

Bellei, C., Canales, M., Orellana, V., y Contreras, M., Elección de escuela en sectores populares: Estado, mercado e integración social. Revista Austral de Ciencias Sociales, 31, 91-110 (2016).

Boekaerts, M., Self-regulated learning: Bridging the gap between metacognitive and metamotivation theories. doi: 10.1207/s15326985ep3004_4, Educational Psychologist, 30(4), 195-200 (1995)

Boekaerts, M., y Cascallar, E., How far have we moved toward the integration of theory and practice in self-regulation? doi: 10.1007/s10648-006-9013-4, Educational Psychology Review, 18(3), 199-210 (2006)

Bourdieu, P., y Passeron, J.C., Los herederos. Los estudiantes y la cultura. $2^{\text {a }}$ edición,1-27. Siglo XXI, Buenos Aires, Argentina (2009).

Boruchovitch, E., y Santos, A.A., Psychometric Studies of the Learning Strategies Scale for University Students. doi: 10.1590/1982-43272560201504, Paidéia (Ribeirão Preto), 25(60), 19-27 (2015)

Bransen, D., y Govaerts, M. J., How to conceptualise self-regulated learning: Implications for measurement. doi:10.1111/medu.14183, Medical Education. 2020, 1-3 (2020)

Canales, M., Bellei, C., y Orellana, V., ¿Por qué elegir una escuela privada subvencionada? Sectores medios emergentes y elección de escuela en un sistema de mercado. doi: 10.4067/S0718-07052016000400005, Estudios pedagógicos, 42(3), 89- 109 (2016)

Cleary, T. J., y Platten, P., Examining the Correspondence between Self-Regulated Learning and Academic Achievement: A Case Study Analysis. doi: 10.1155/2013/272560, Education Research International, 23, 1-18 (2013)

Daura, F. T., Aprendizaje autorregulado e intervenciones docentes en la universidad. doi: 10.15517/revedu.v41i2.21396, Revista Educación, 41(2), 1-19 (2017)

Díaz, A., Pérez, M. V., y otros dos autores, Impacto de un entrenamiento en aprendizaje autorregulado en estudiantes universitarios. Perfiles Educativos, 39(157), 87-104 (2017) 
Díaz, A., Pérez, M.V., y otros cuatro autores. Procesos de autorregulación del aprendizaje en estudiantes universitarios de primer año. International Journal of Developmental an Educational Psychology, 4(1), 789-800 (2010)

Elvira-Valdés, M. A., y Pujol, L., Autorregulación y rendimiento académico en la transición secundariauniversidad. doi:10.11600/169271525, Revista Latinoamericana de Ciencias Sociales, Niñez y Juventud, 10(1), 367-378 (2012)

Fernández, E., Bernardo, A., y otros cuatro autores, Prediction of the use of self-regulation strategies in higher education. doi: 10.6018/analesps.29.3.139341, Anales de psicología, 29(3), 865-875 (2013)

Hernández, A. y Camargo, Á., Adaptation and validation of the Inventory of Self-Regulation Strategies in university students. doi: 10.1016/j.sumpsi.2017.02.001, Suma Psicológica, 24(1), 9-16 (2017a).

Hernández, A., y Camargo, Á., Self-regulation of learning in higher education in Ibero-America: a systematic review. doi: 10.1016/j.rlp.2017.01.001, Revista Latinoamericana de Psicología, 49(2), 146-160 (2017b)

$\mathrm{Hu}$, L., y Bentler, P., Cutoff criteria for fit indexes in covariance structure analysis: Conventional criteria versus new alternatives. doi: 10.1080/10705519909540118, Structural Equation Modeling. A Multidisciplinar Journal, 6(1), 1-55 (1999)

Mega, C., Ronconi, L., y De Beni, R., What makes a good student? How emotions, self-regulated learning, and motivation contribute to academic achievement. doi: 10.1037/a0033546, Journal of Educational Psychology, 106(1), 121-131 (2014)

Peeters, J., De Backer, F., y otros cuatro autores, The Role of Tachers' Self-regulatory Capacities in the Implementation of Self-regulated Learning Practices. doi: 10.1016/j.sbspro.2014.01.504, Procedia - Social and Behavioral Sciences, 116, 1963-1970 (2014)

Pintrich, P.R., y De Groot, E.V., Motivational and Self-Regulated Learning Components of Classroom Academic Performance. doi: 10.1037/0022-0663.82.1.33, Journal of Educational Psychology, 82(1), 33-40 (1990)

Pool-Cibrián, W.J., y Martínez-Guerrero, J.I., Autoeficacia y uso de estrategias para el aprendizaje autorregulado en estudiantes universitarios. Revista Electrónica de Investigación Educativa, 15(3), 21-37 (2013)

Streiner, D. L., Norman, G. R., y Cairney, J., Health measurement scales: a practical guide to their development and use. Oxford University Press, Oxford, Inglaterra (2015)

Valenzuela, J. P., Bellei, C., y otro autor, Socioeconomic school segregation in a market-oriented educational system. The case of Chile. doi: 10.1080/02680939.2013.806995, Journal of education Policy, 29(2), 217-241 (2014)

Winne, P. H., A metacognitive view of individual differences in self-regulated learning. doi: 10.1016/S1041-6080(96)900229, Learning and Individual Differences, 8(4), 327-353 (1996)

Zambrano, C., Autoeficacia, Prácticas de Aprendizaje Autorregulado y Docencia para fomentar el Aprendizaje Autorregulado en un Curso de Ingeniería de Software. doi: 10.4067/S0718-50062016000300007, Formación Universitaria, 9(3), 51-60 (2016)

Zambrano, C., Rojas, D.F., y otros dos autores, Propiedades Psicométricas del Inventario de Estrategias de Autorregulación en Estudiantes de Pedagogía Chilenos. doi:10.4067/S0718-50062018000500085, Formación Universitaria, 11(5), 85-92 (2018)

Zambrano-Matamala, C., Rojas-Diaz, D.F., y otros tres autores, Perception of student-teachers regarding self-regulated learning. Pedagogy in Basic and Higher Education-Current Developments and Challenges. 1ª edición, 1-20. IntechOpen, Londres, Inglaterra (2019)

Zimmerman, B. J., A social cognitive view of self-regulated learning, doi: 10.1037/0022-0663.81.3.329, Journal of Educational Psychology, 81(3), 329-339 (1989) 
